\title{
Hernia of the hypogastric linea alba without hernia sac: evidence supporting the vascular lacunae hypothesis
}

\author{
Tianhao Xie $\cdot$ Xiangxiang Ren $\cdot$ Chao Zhang $\cdot$ Xiaoshi Jin
}

Received: 17 August 2020 / Accepted: 19 August 2020 / Published online: 8 October 2020

(C) The Author(s) 2020

To the editor,

Hernias of the linea alba are relatively rare and most often detected in the midsection abdominal wall. According to the definitions of the European Hernia Society (EHS) and the American Hernia Society (AHS), a hernia where the center of the defect is in the midline above the umbilicus up to the xiphoid process is referred to as an "epigastric hernia" [1]. Notwithstanding this definition, it is well-known that the linea alba has another portion, extending from the umbilicus to the symphysis pubis; however, the EHS and AHS do not describe hernias that occur on the linea alba below the umbilicus. The vascular lacunae hypothesis suggested by Moschowitz is widely accepted as a possible explanation for the pathogenesis of linea alba hernias in the upper abdomen [2]. Here, we present an unusual case of linea alba hernia without a hernia sac, located in the hypogastric abdominal wall and through surgery show that blood vessels traversed the hernia ring.

A 64-year-old woman was referred to our Department of Surgery in October 2019, complaining of an asymptomatic lump shaped like a quail egg in the middle of the hypogastric abdominal wall that had been present for 20 years. She had experienced pain in her lower abdomen when she bent down for the previous 2 weeks, which was relieved by standing.

Computed tomography (CT) revealed a local defect $($ size, $0.7 \mathrm{~cm})$ in the linea alba of the lower ab-

T. Xie $\cdot$ X. Ren $\cdot$ X. Jin $(\bowtie)$

Department of General Surgery, Affiliated

Hospital of Hebei University, 212 East Yuhua

Road, 071000 Baoding, Hebei, China

doctorjinxiaoshi@126.com

\section{Zhang}

Department of Surgery, Li County Hospital of Traditional Chinese Medicine, Baoding, Hebei, China domen with abdominal fat entering the subcutaneous fat layer through the defect (Fig. 1). Linea alba hernia was confirmed during surgery. There was no hernia sac, only a portion of the median umbilical ligament was embedded into the hernia ring and it was difficult to pull back into the abdominal cavity. We removed this section of the median umbilical ligament and observed a hernia ring $(1.0 \mathrm{~cm}$ in diameter $)$ in the linea alba. The median umbilical ligament and its accompanying blood vessels traversed the hernia ring (Fig. 2). The linea alba defect and peritoneum were sutured, and the hernia was repaired using the intraperitoneal onlay mesh (IPOM) technique with an intraperitoneal preformed patch.

The patient's abdominal pain disappeared after the operation.

Although the vascular lacunae hypothesis is generally accepted as describing a mechanism that can underlie hernias of the linea alba, only three cases supported this hypothesis [2, 3]. The outer and inner surfaces of the linea alba above the umbilicus have different structural characteristics [4]. On the outer surface, the intersected peritoneal fibers are of uniform thickness and closely interwoven, with small holes passing through, which contain small blood vessels and nerve branches; however, large holes are rarely seen. On the inner surface, the aponeurotic fiber bundle is uneven in thickness, often forming a thick bundle or plate, and the distribution is somewhat irregular. There are holes or cracks passing through, which include nerves and vascular branches, that result in defects in the inner layer of the linea alba.

Here, we report a rare case of a hypogastric linea alba hernia, where accompanying blood vessels traversed the hernia ring. To our knowledge, publications of this type of linea alba hernia are rare. Our case provides evidence in support of the vascular lacunae hypothesis. 


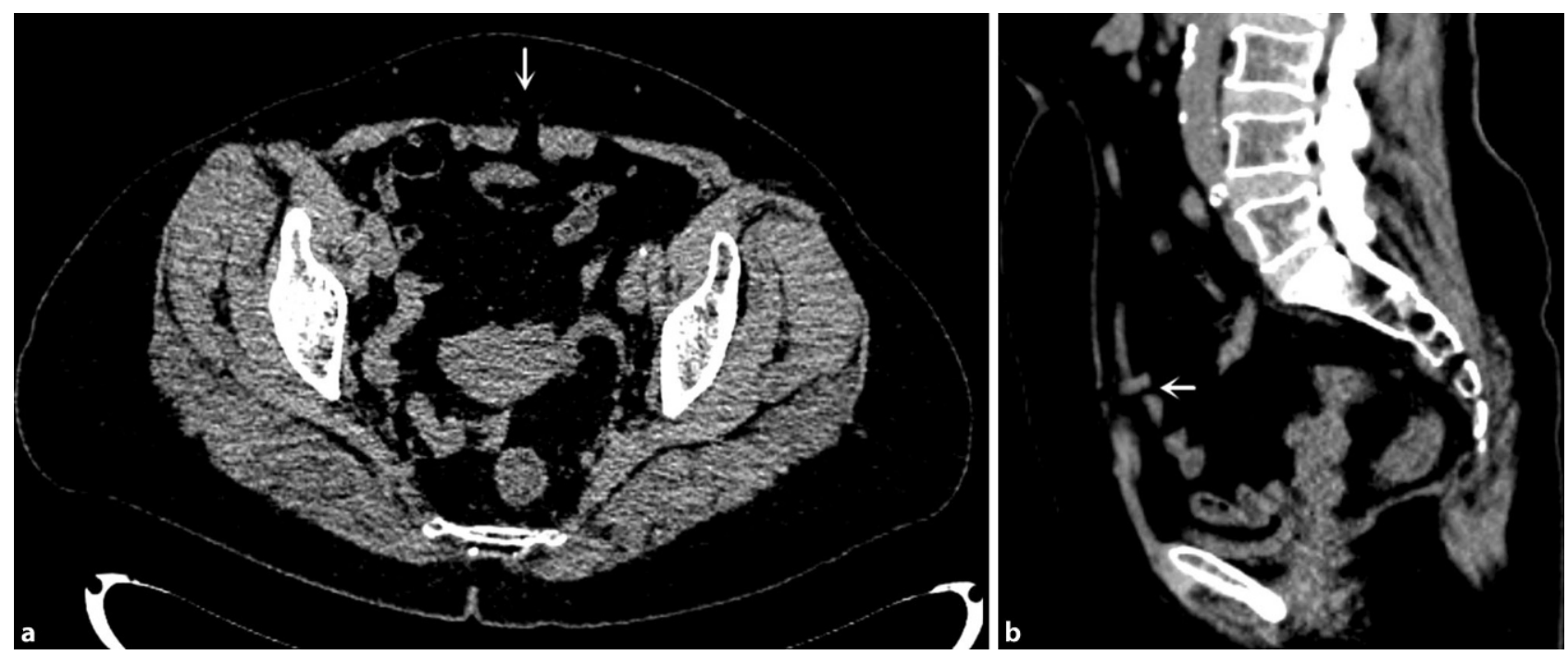

Fig. 1 Computed tomography images of a local $0.7-\mathrm{cm}$ defect in the linea alba of the lower abdomen and abdominal fat entering the subcutaneous fat layer through the defect (white arrow)
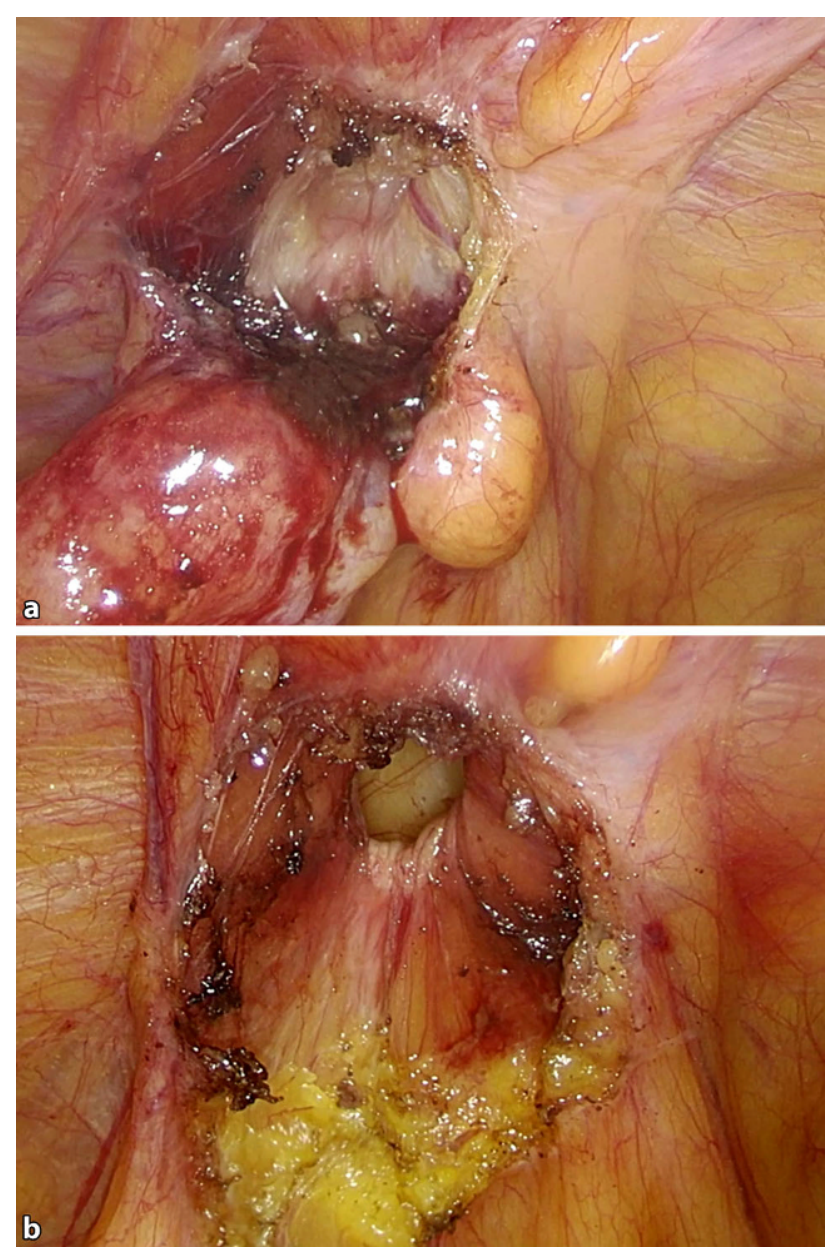

Fig. 2 The median umbilical ligament and its accompanying blood vessels traversing the hernia ring
Funding This article was supported by grants from the Key Research and Development Program of HeibeiProvince (202030701180172), and Science Technology Plan of Baoding Municipal Department of Science and Technology (1951ZF091 and 2041ZF172).

Conflict of interest T.H. Xie, X.X. Ren, C. Zhang and X.S. Jin declare that they have no competing interests.

Open Access This article is licensed under a Creative Commons Attribution 4.0 International License, which permits use, sharing, adaptation, distribution and reproduction in any medium or format, as long as you give appropriate credit to the original author(s) and the source, provide a link to the Creative Commons licence, and indicate if changes were made. The images or other third party material in this article are included in the article's Creative Commons licence, unless indicated otherwise in a credit line to the material. If material is not included in the article's Creative Commons licence and your intended use is not permitted by statutory regulation or exceeds the permitted use, you will need to obtain permission directly from the copyright holder. To view a copy of this licence, visit http://creativecommons.org/licenses/by/4.0/.

\section{References}

1. Henriksen NA, Montgomery A, Kaufmann R, et al. Guidelines for Treatment of umbilical and epigastric hernias from the European Hernia Society and Americas Hernia Society. Br J Surg. 2020;107(3):171-90.

2. Moschcowitz AV. Epigastric hernia without palpable swelling. Ann Surg. 1917;66:300-7.

3. Lang B, Lau H, Lee F. Epigastric hernia and its etiology. Hernia. 2002;6:148-50.

4. Askar OM. Aponeurotic hernias. Recent observations upon paraumbilical and epigastric hernias. Surg Clin North Am. 1984;64:315-33.

Publisher's Note Springer Nature remains neutral with regard to jurisdictional claims in published maps and institutional affiliations. 